\title{
Biodiversity of Intertidal Estuarine Fungi on Phragmites at Mai Po Marshes, Hong Kong
}

\author{
M. O. K. Poon and K. D. Hyde* \\ Department of Ecology and Biodiversity, The University of Hong Kong, Pokfulam Road, Hong Kong \\ * Corresponding author
}

Intertidal decaying stems and leaf sheaths of Phragmites australis were randomly collected and their mycota examined. Sixty one species of fungi were associated with the decaying stems and leaf sheaths, including Antiptodera spp., Lignincola laevis, Phomatospora phragmiticola and Zopfiella latipes. The following new species are described, Halosarpheia phragmiticola, Massarina phragmiticola, Phomatospora phragmiticola and Cytoplacosphaeria phragmiticola. The fungal communities associated with decaying Phragmites australis permanently submerged in the gei wai (tidal shrimp farms) differ from those in the intertidal region. The diversity of these fungi are discussed in relation to the biodiversity of fungi in mangrove communities in Hong Kong and with those fungi of other salt marsh communities.

\section{Introduction}

Temperate coastal wetlands are often dominated by salt marsh grasses, such as species of Spartina and Juncus. Most research on the associated mycota of these temperate plants in based on Spartina (Johnson and Sparrow 1961, Meyers et al. 1970, Meyers 1974, Gessner 1976, 1977, Gessner and Kohlmeyer 1976, Kohlmeyer and Kohlmeyer 1979). There is also some information on the fungi associated with Juncus roemerianus Scheele (Kohlmeyer and VolkmannKohlmeyer 1993 a, b, 1995, Volkmann-Kohlmeyer and Kohlmeyer 1993, 1994, Kohlmeyer et al. 1995 a, b, c, 1966).

Mangrove vegetation is the tropical counterpart of temperate tidal salt marshes (Kohlmeyer and Kohlmeyer 1979). There is a large body of information on mangrove fungi, with most data on biodiversity (Hyde and Jones 1988, Hyde and al. 1986, 1992, 1993, Hyde 1992, Hyde and Nakagiri 1992, Jones and Agerer 1992, Sadaba et al. 1995).

In subtropical regions, salt marsh grasses such as Phragmites australis (Cav.) Trin. ex Steud [also known as Phragmites communis (L.) Trin.] coexist with the mangrove tree species. The tidal shrimp ponds (gei wai) of Mai Po were originally excavated from native mangrove habitats with communities of Kandelia candel (L.) Druce, Avicennia marina (Forsk.) Vierh. and Aegiceras corniculatum (L.) Blanco. Traditional gei wais at Mai Po are characterised by repeated draining and flooding and details of operational methods can be found in Macintosh (1983). Mangroves (mostly Kandelia candel) were originally the chief primary producers in Mai Po Marshes. However, due to progressive change in the operation of the gei wais from traditional shrimp catching to fish culture, this has resulted in some gei wais (e.g. gei wai no. 12) being kept undrained for several months. Semi-permanent flooding had resulted in death of mangroves which are subsequently replaced by Phragmites australis, which colonises the banks and edges of gei wais and elevated mud platforms. Phragmites australis has now acquired co-dominance in the gei wais with Kandelia candel, and is one of the main primary producers (Lee 1990). It was estimated that about $40 \%$ of open or elevated mud platforms in the gei wais were covered with $P$. australis in early 1988 (Lee 1990).

Fungi have long been recognised as one of the major decomposers of salt marsh plants, beside bacteria (e.g. Meyers et al. 1970, Gessner 1977, Newell 1993, 1996). Recent research on the dynamics of microorganisms and the change in the chemical composition of decomposing Phragmites australis in Japan, have shown that fungi are the main decomposers of decaying leaves before and after submergence in seawater, while bacteria were only dominant decomposers within the short period following submergence (Tanaka 1991). Tanaka (1991) suggested that the decrease in the fungal populations during the short time following submergence can be attributed to the inability of terrestrial fungi to survive in the saline aquatic environment.

As crucial decomposers of a major primary producer, in a highly productive subtropical estuarine region, fungi warrant more attention to their biodiversity and ecology than has previously been given. Unfortunately, the mycota of Phragmites australis, is even less well investigated than either Spartina alterniflora Loisel. or Juncus roemerianus. Only two marine fungi, Phaeosphaeria albopunctata (West.) Shoemaker et C. E. Babc. and Cirrenalia fusca I. Schmidt, have been reported from $P$. australis by previous re- 
searchers (Kohlmeyer and Kohlmeyer 1979). Massariosphaeria typhicola (Karst.) Leucht. has been found on $P$. australis in freshwater locations in Finland (Karsten 1873). Several terrestrial mitosporic fungi have also been reported on leaves and stems and leaf sheaths of P. australis (e. g. Cunnell 1958, Sutton and Alcorn 1974). In this study an assessment of the biodiversity of the mycota found on decaying stems and leaf sheaths of $P$. australis at the Mai Po Marshes is made.

\section{Materials and Methods}

\section{Study area}

All sampling was conducted along the edge of a tidal shrimp pond ('gei wai', no. 12) and on both sides of the sluice gate of gei wai no. 12 outside the security fence, in Mai Po Marshes. Mai Po Marshes are situated along the northwestern coast of the New Territories facing the Pearl River estuary $\left(22^{\circ} 29^{\prime} \mathrm{N}\right.$, $\left.114^{\circ} 02^{\prime} \mathrm{E}\right)$.

\section{Sampling site}

Two sampling sites were chosen to initiate a comparison between the fungi found in two different habitats.

Outside security fence: Phragmites australis is subjected to natural tidal inundation with the basal portion (ca. $30 \mathrm{~cm}$ ) of the plant submerged regularly during high tides.

Along the edge of gei wai: The amount of time for the basal to middle portion of plant being inundated is regulated solely by the operation method of gei wai. During the period of this study, up to one half (ca. $70 \mathrm{~cm}$ ) of the plant had been immersed for several months.

\section{Collection of samples}

In order to collect saprophytic fungi responsible for the decompositions of decaying Phragmites australis, dead stems and leaf sheaths were randomly collected from within one to two meters of the shore at the two sites. These were lying on the mangrove floor outside the security fence, or standing upright with the lower part submerged in the gei wai, and were naturally decomposing samples. One hundred and twenty five decaying stem and leaf sheath segments (ca. $15 \mathrm{~cm}$ ) were collected from outside the security fence and 144 samples were collected from the gei wai, with ca. half of the samples collected in August and the other half in November 1995. Samples were placed in plastic bags, sealed and returned to the laboratory. Samples were then incubated on moist tissue paper, in clear plastic boxes $(25 \times 12 \times 10 \mathrm{~cm})$ at room remperature and normal lighting conditions, for what was found to be an optimum period of $1-3$ weeks to induce sporulation of fungi. The fungi were identified by using a Leica MZ 12 dissecting microscope at 15-20 $\times$ maghification. Voucher slides and/ or dried material of the fungi found were prepared and are held in the mycological herbarium of The University of Hong Kong [HKU(M)].

\section{Analyses}

Frequency of occurrence of fungi collected is expressed as the number of collections of a species at each site divided by the total number of samples examined from this site. Based on these figures, fungi collected are classified as 'very frequent' $(>20 \%)$, 'fequent' $(10-20 \%)$ and 'infrequent' $(<10 \%)$ species, as adopted by Tan and Leong (1989). It was not feasible to identify all taxa to species level, as some genera lack modern treatments or up to date keys and may contain numerous species. However, each unique taxon is named (e.g. Farrowia sp. represents one species of Farrowia, Phomopsis sp. 1 and Phomopsis sp. 2 represent two species of Phomopsis. They are reported here in order to give an estimation of fungal diversity occurring on Phragmites australis at Mai Po Marshes.

Water temperatures at the time of collection was between $24-31{ }^{\circ} \mathrm{C}$, while the salinity range outside security fence and along edge of gei wai no. 12 was 2.5-19\%o, and 2-15\%o respectively. In situ ammonium concentration $\left(\mathrm{NH}_{4}^{+}\right)$of water outside security fence and along edge of gei wai no. 12 was $15-20$ ppm, and 22-26 ppm respectively.

\section{Results}

Sixty one taxa of higher fungi were found associated with decaying stems and leaf sheaths of Phragmites australis and the results are presented in Table I. Forty one taxa were collected from 125 decaying plant samples from outside security fence, and 47 taxa were found on 144 plant samples along the edge of the gei wai. The ratio of ascomycetes to coelomycetes to hyphomycetes was roughly equal $(17: 19: 25)$. Lignincola laevis was overall the most common species $(22.7 \%)$, while another very frequent taxon was Colletotrichum sp. (21.2\%). Frequent taxa were Phomopsis sp. 1 (19.3\%), Aniptodera phragmiticola (15.2\%), Fusarium sp. (14.9\%), Cladosporium sp. (13\%), Trichoderma sp. (12.3\%), Cytoplea sp. (11.9\%) and Rhinocladiella $\mathrm{sp} .(11.2 \%)$, while other taxa were infrequent.

Outside the security fence, Lignincola laevis (37.6\%) was the most common species. Other very frequent taxa were Trichoderma sp. (26.4\%), Antiptodera phragmiticola $(23.2 \%)$ and Collectotrichum sp. (22.4\%). Along the edge of the gei wai, Fusarium sp. $(28.8 \%)$, Cladosporium sp. (24.3\%), Phomopsis sp. 1 (21.5\%) and Colletotrichum sp. (20.1\%) were very frequent.

Twenty seven taxa including Colletotrichum sp., Phomopsis sp. 1, Rhinocladiella sp., Lignincola laevis and Aniptodera phragmiticola were found common to 
both sites. The majority of taxa (34), however, occurred at a single site. Thirteen taxa were found only outside the security fence, such as Trichoderma sp., Phomatospora marina and Phragmitensis marina. Twenty taxa were also found only along the edge of the gei wai, such as Fusarium sp., Cladosporium sp. and Macrophomina sp.

\section{Saprophytic fungi occurring on Phragmites australis}

The lower parts of the standing decaying stems and leaf sheaths (up to $30 \mathrm{~cm}$ ) of Phragmites australis found outside the security fence, are subjected to daily tidal inundation. Along the edge of the gei wai, up to one half of the standing decaying stems and leaf sheaths of $P$. australis $(\mathrm{ca} 70 \mathrm{~cm})$ are immersed for several months. Fungi occurring on these lower plant parts can be considered to be intertidal fungi, while those occurring on the upper parts of the stems and leaf sheaths of $P$. australis are terrestrial. Middle portions of the plants are occasionally submerged or exposed to salt spray and hence colonised by mostly intertidal fungi.

Intertidal fungi. Differences in intertidal fungal composition between the two sites (outside the security fence and along the edge of the gei wai) were observed. Eleven intertidal fungi were confined to the decaying stems and leaf sheaths lying on the mangrove floor, collected outside the security fence. Lignincola laevis (37.6\%) and Aniptodera phragmiticola $(23.2 \%)$ were the most dominant intertidal species here, while Gaeumannomyces sp. was an infrequent ascomycete. Three coelomycetes (Chaetasbolisia sp., Microsphaeropsis sp., Stauronema sp.) and two hyphomycetes (Pithomyces maydiscus and Spegazzinia tessarthra) were infrequent.

Six hyphomycetes (Acremonium sp. 2, Alternaria alternata, Arthrobotrys conoides, Dactylaria sp., Gliomastix sp. 1, Sarocladium sp.) were confined to the plant samples collected at the water/air interface, along the side of the gei wai. The most common taxa being the hypohomycetes Cladosporium sp. (24.3\%), Sarocladium sp. (8.3\%) and Arthrobotrys conoides (4.9\%). One coelomycete, Dinemasporium strigosum, and one ascomycete, Massarina thalassiae also occurred on these lower portions of plant samples.

Most intertidal fungi were found at both sites, although some exhibited a clear affinity towards a specific site. The ascomycetes Aniptodera phragmiticola, Lignincola laevis and Massarina phragmiticola were mostly found outside the security fence. The coelomycetes Cytoplascosphaeria phragmiticola, Phoma sp., Phomopsis sp. 1, Sclerostagonospora sp. and Septoriella sp.) were common to both sites, although Cytoplacosphaeria phragmiticola and Septoriella sp. were more common along the edge of the gei wai. The hyphomycetes Arthrinium state of Apiospora sp., Drechslera hawaiiensis, Phaeoisaria sp., Rhinocladiella sp., Stachybotrys sp., and Tetraploa aristata were also common to both sites, but Tetraploa aristata was more frequently recovered outside the security fence.

Terrestrial fungi. Sixteen terrestrial fungi were found associated with the decomposition of the apical to middle portion of decaying stems and leaf sheaths of Phragmites australis. Four of these were common to both sites. Chaetomium globosum was the only terrestrial ascomycete found on the upper levels of the plant samples. This species was common at both sites, but was more frequently recovered outside the security fence. Cytoplea sp. and the Arthrinium state of Apiospora montagnei were found on plant samples, both outside the security fence and along the edge of the gei wai.

Outside the security fence, Trichoderma sp. (26.4\%) and Chaetomium globosum (6.4\%) were the most frequently recorded terrestrial fungi. Phomopsis sp. 2 , Tetranacrium sp., Dendrostilbella sp. and Trichoderma sp. were only collected here.

Along the edge of the gei wai, Fusarium sp. (28.8\%) was the most commonly recorded taxon. Eight less frequent terrestrial deuteromycetes (Cladosporium $\mathrm{sp.}$ Macrophomina sp. Neottiosporina sp. Pestalotiopsis sp. Stagonospora sp. Acremonium sp. 1, Gliomastrix sp. 2 and Paecilomyces sp.) were limited to plant samples along the edge of the gei wai.

\section{Descriptions of fungi}

An account of selected saprophytic fungi found on decaying stems and leaf sheaths of Phragmites australis during this study is given in the following section.

\section{Ascomyetes}

Halosarpheia phragmiticola O. K. Poon et K. D. Hyde, sp. nov.

Figs $1-10$

Etymology: In reference to Phragmities australis the host.

\section{Diagnosis}

Ascomata ca $350 \mu m$ in diameter., ca $400 \mu m$ alta, globosa vel subglobosa, immersa vel superficialia, ostiolata, papillata, coriacea, nigra, solitaria. Asci 105$167.5 \times 32.5-47.5 \mu \mathrm{m}$, 8-spori, clavati, pedicellati, apparatu apicali praediti. Ascosporae 25-35 × 7.7$10.5 \mu \mathrm{m}$, ellipsoido-fusiformes, 1-septatae, appendiculatae.

Ascomata $350-400 \mu \mathrm{m}$ in diameter, globose to subglobose, immersed to superficial, ostiolate, coriaceous, black, solitary, with a long black cylindrical neck (Fig. 1). Neck up to $700 \mu \mathrm{m}$ long, $70 \mu \mathrm{m}$ in diameter, cylindrical, superficially covered with short hyaline hairs, periphysate. Peridium up to $10 \mu \mathrm{m}$ thick, two layered; outer layer comprising black thick-walled cells; inner layer comprising hyaline 
Table I. Estuarine saprophytic fungi associated with decaying stems and leaf sheaths of Phragmites australis collected in Mai Po Marshes in August and November 1995.

\begin{tabular}{|c|c|c|c|c|c|c|}
\hline \multirow[t]{2}{*}{ Fungi } & \multicolumn{2}{|c|}{ Outside fence } & \multicolumn{2}{|c|}{$\begin{array}{l}\text { Along edge of } \\
\text { gei wai }\end{array}$} & \multirow{2}{*}{$\begin{array}{l}\text { Total } \\
\text { no. of } \\
\text { collec- } \\
\text { tions }\end{array}$} & \multirow{2}{*}{$\begin{array}{l}\% \\
\text { occur- } \\
\text { rence }\end{array}$} \\
\hline & $\begin{array}{l}\text { No. of } \\
\text { collec- } \\
\text { tions }\end{array}$ & $\begin{array}{l}\% \\
\text { occur- } \\
\text { rence }\end{array}$ & $\begin{array}{l}\text { No. of } \\
\text { collec- } \\
\text { tions }\end{array}$ & $\begin{array}{l}\% \\
\text { occur- } \\
\text { rence }\end{array}$ & & \\
\hline
\end{tabular}

\section{ASCOMYCETES (17 taxa)}

Aniptodera chesapeakensis Shearer et Miller Aniptodera phragmiticola O. K. Poon et K. D. Hyde

Chaetomium globosum Kunze ex Steud.

Farrowia sp.

Gaeumannomyces sp.

Halosarpheia unicaudata (E. B. G. Jones et

LeCampion-Alsumard) R. G. Johnson, E. B. G.

Jones et S. T. Moss ex Kohlm. et Volkm.-Kohlm.

Leptosphaeria sp.

Lignincola laevis Höhnk

Massarina phragmiticola O. K. Poon et K. D. Hyde

Massarina thalassiae Kohlm. et Volkm.-Kohlm.

Nectria haematococca Berk. et Broome

Phomatospora phragmiticola O. K. Poon et K. D. Hyde

Phragmitensis marina M. Wong, O. K. Poon et K. D. Hyde

Pleospora spartinae (Webster et Lucas) Apinis et Chesters

Pseudohalonectria falcata Shearer

Verruculina enalia (Kohlm.) Kohlm. et Volkm.-Kohlm.

Zopfiella latipes (Lundqvist) Malloch et Cain

\section{DEUTEROMYCETES (44 species)}

Coelomycetes (19 species)

Chaetasbolisia sp.

Chaetospermum camelliae Agnihothr.

Colletotrichum sp.

Cytoplacosphaeria phragmiticola O. K. Poon et K. D. Hyde

Cytoplea sp.

Dinemasporium strigosum Pers. ex Fr.

Macrophomina sp.

Microsphaeropsis sp.

Neottiosporina sp.

Pestalotiopsis sp.

Phoma sp.

Phomopsis sp. 1

Phomopsis sp. 1

Pseudorobillarda phragmitis (Cunnell) Morelet

Sclerostagonospora sp.

Septoriella sp.

Stagonospora sp.

Stauronema sp.

Tetranacrium sp.

$\begin{array}{rlr}6 & 4.8 & 2 \\ 29 & 23.2 & 12 \\ 8 & 6.4 & \\ 0 & 0 & \\ 1 & 0.8 & 0 \\ 0 & 0 & 13\end{array}$

2
12
1
1
0
13

$\begin{array}{lrr}1.4 & 8 & 3 \\ 8.3 & 41 & 15.2 \\ 0.7 & 9 & 3.3 \\ 0.7 & 1 & 0.4 \\ 0 & 1 & 0.4 \\ 9 & 13 & 4.8\end{array}$

$\begin{array}{rll}1 & 0.8 & 8 \\ 47 & 37.6 & 14 \\ 11 & 8.8 & \\ 0 & 0 & \end{array}$

5.6

9.7

0.7

0.7

1.6

$\begin{array}{rr}2 & 1.6 \\ 20 & 15.9\end{array}$

\section{0}

3.2

0

4

1

0.8

12

8.3

13

4.8

Hyphomycetes (25 species) 
Table I. Continued.

\begin{tabular}{|c|c|c|c|c|c|c|}
\hline \multirow[t]{2}{*}{ Fungi } & \multicolumn{2}{|c|}{ Outside fence } & \multicolumn{2}{|c|}{$\begin{array}{l}\text { Along edge of } \\
\text { gei wai }\end{array}$} & \multirow{2}{*}{$\begin{array}{l}\text { Total } \\
\text { no. of } \\
\text { collec- } \\
\text { tions }\end{array}$} & \multirow{2}{*}{$\begin{array}{l}\% \\
\text { occur- } \\
\text { rence }\end{array}$} \\
\hline & $\begin{array}{l}\text { No. of } \\
\text { condi- } \\
\text { tions }\end{array}$ & $\begin{array}{l}\% \\
\text { occur- } \\
\text { rence }\end{array}$ & $\begin{array}{l}\text { No. of } \\
\text { collec- } \\
\text { tions }\end{array}$ & $\begin{array}{l}\% \\
\text { occur- } \\
\text { rence }\end{array}$ & & \\
\hline Alternaria alternata Nees & 0 & 0 & 1 & 0.7 & 1 & 0.4 \\
\hline Arthrinium state of Apiospora sp. & 5 & 4 & 8 & 5.6 & 13 & 4.8 \\
\hline Arthrinium state of Apiospora montagnei Sacc. & 11 & 8.8 & 8 & 5.6 & 19 & 7.1 \\
\hline Arthrobotrys sp. & 16 & 12.8 & 3 & 2.1 & 19 & 7.1 \\
\hline Arthrobotrys conoides Drechsler & 0 & 0 & 7 & 4.9 & 7 & 2.6 \\
\hline Cladosporium sp. & 0 & 0 & 35 & 24.3 & 35 & 13 \\
\hline Dactylaria sp. & 0 & 0 & 11 & 7.6 & 11 & 4.1 \\
\hline Dendrostilbella sp. & 3 & 2.4 & 0 & 0 & 3 & 1.1 \\
\hline $\begin{array}{l}\text { Drechslera hawaiiensis (Bugnicourt) Subram. et } \\
\text { Jain ex M. B. Ellis }\end{array}$ & 2 & 1.6 & 2 & 1.3 & 4 & 1.4 \\
\hline Ellisembia sp. & 0 & 0 & 4 & 2.8 & 4 & 1.5 \\
\hline Fusarium sp. & 0 & 0 & 40 & 28.8 & 40 & 14.9 \\
\hline Gliomastix sp. 1 & 3 & 2.4 & 9 & 6.3 & 12 & 4.5 \\
\hline Gliomastix sp. 2 & 0 & 0 & 5 & 3.5 & 5 & 1.9 \\
\hline Paecilomyces sp. & 0 & 0 & 6 & 4.2 & 6 & 2.2 \\
\hline Penicillium sp. & 9 & 7.2 & 3 & 2.1 & 12 & 4.5 \\
\hline Phaeosiaria sp. & 1 & 0.8 & 3 & 2.1 & 4 & 1.4 \\
\hline Pithomyces maydicus (Sacc.) M. B. Ellis & 3 & 2.4 & 0 & 0 & 3 & 1.1 \\
\hline Rhinocladiella sp. & 13 & 10.4 & 17 & 11.8 & 30 & 11.2 \\
\hline Sarocladium sp. & 0 & 0 & 12 & 8.3 & 12 & 4.5 \\
\hline Spegazzinia tessarthra (Berk. et Curt.) Sacc. & 1 & 0.8 & 0 & 0 & 1 & 0.4 \\
\hline Stachybotrys sp. & 4 & 3.2 & 2 & 1.3 & 6 & 2.2 \\
\hline Tetraploa aristata Berk. et Broome & 14 & 11.2 & 1 & 0.7 & 15 & 5.6 \\
\hline Trichoderma sp. & 33 & 26.4 & 0 & 0 & 33 & 12.3 \\
\hline No. of samples examined & 125 & & 144 & & 269 & \\
\hline
\end{tabular}

thin-walled cells, both in the form of textura angularis (Fig. 2). Catenophyses 5-15 $\times 27.5-145 \mu \mathrm{m}$ septate, hyaline, developing from pseudoparenchyma of the centrum. Asci 105-167.5 $\times 32.5-47.5 \mu \mathrm{m}$, 8spored, clavate, pedicellate, with an apical pore, IKInegative, thin-walled, unitunicate, cytoplasm constricted below the apex, persistent, maturing successively on the ascogenous tissue at base of locule (Figs 3-8). Ascospores 25-35 $\times 7.7-10.5 \mu \mathrm{m}$, ellipsoidfusiform, 1-septate, not constricted at septa, provided with a large lipid guttule in each cell, bearing one appendage at each end; appendages hamate, filamentous, hyaline (Figs 9, 10).

Mode of life: Saprobic.

Habitat: Stems and leaf sheaths of Phragmites australis.

\section{Known distribution: Hong Kong.}

Material examined: Decaying stems and leaf sheaths of intertidal $P$. australis, Mai Po Marshes, Hong Kong $\left(22^{\circ} 29^{\prime} \mathrm{N}, 114^{\circ} 02^{\prime} \mathrm{E}\right), 17$ Aug. 1995, O. K. Poon [HKU(M) 5186, holotype]; decaying stems and leaf sheaths of intertidal $P$. australis, Mai Po Marshes, Hong Kong $\left(22^{\circ} 29^{\prime} \mathrm{N}, 114^{\circ} 02^{\prime} \mathrm{E}\right), 16$ Nov. 1995, O. K. Poon [HKU(M) 5187].
This collection on decaying stems and leaf sheaths of $P$. australis in the intertidal region is superficially very similar to Halosarpheia culmiperda Kohlm., Volkm.-Kohlm. et O. E. Erikss. (Kohlm. et al. $1995 \mathrm{c})$. The species, however differs, as H. culmiperda has wider ascospores $(9-13 \mu \mathrm{m})$ with subapical cap-like appendages, narrower clavate asci $(23.5-30$ $\mu \mathrm{m}$ wide) and tawny ascomata. In Halosarpheia phragmiticola ascospores are 7.7-10.5 $\mu \mathrm{m}$ wide, appendages are large and hamate, asci are saccate-clavate and 32.5-47.5 $\mu \mathrm{m}$ wide, and ascomata are black. Halosarpheia phragmiticola should be compared with Aniptodera juncicola Volkm.-Kohlm. et Kohlm., from Juncus roemerianus (VolkmannKohlmeyer and Kohlmeyer 1994). Aniptodera juncicola lacks appendaged ascospores, and has greyish brown to fuscous ascomata. The ascopores also differ in shape from those of Halosarpheia viscosa (I. Schmidt) Shearer et Crane.

Massarina phragmiticola O. K. Poon et K. D. Hyde, sp. nov.

Figs $11-20$

Etymology: In reference to the host Phragmites australis. 


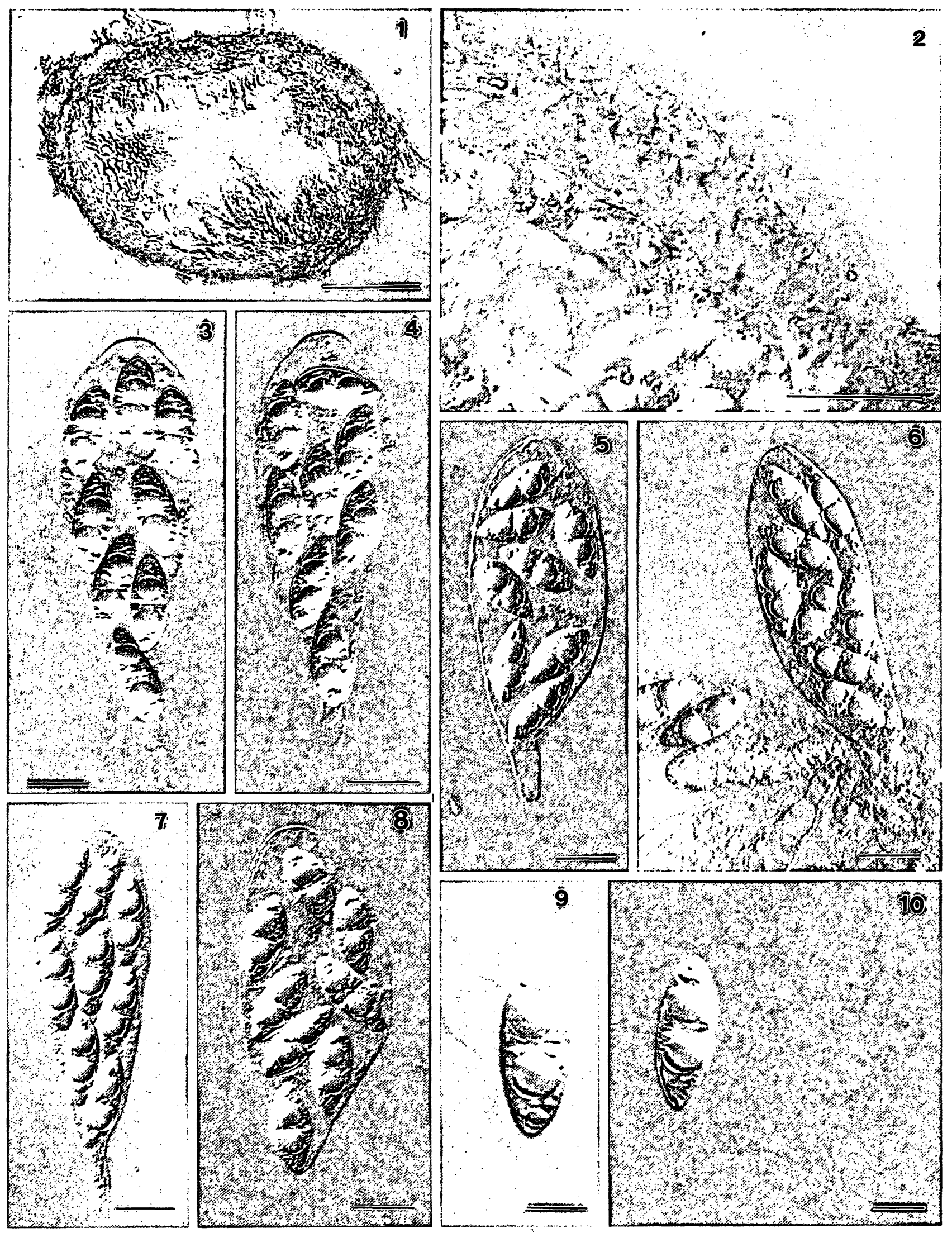

Figs 1-10. Aniptodera phragmiticola (from holotype).

Fig. 1. Section of ascoma (bar $=100 \mu \mathrm{m})$. Fig. 2. Section of ascoma, illustrating peridium (bar $=10 \mu \mathrm{m})$. Figs $3-8$. Mature asci $($ bars $=20 \mu \mathrm{m})$. Figs $9-10$. Mature ascospores with filamentous appendages at both ends $(\mathrm{bars}=10 \mu \mathrm{m})$. 


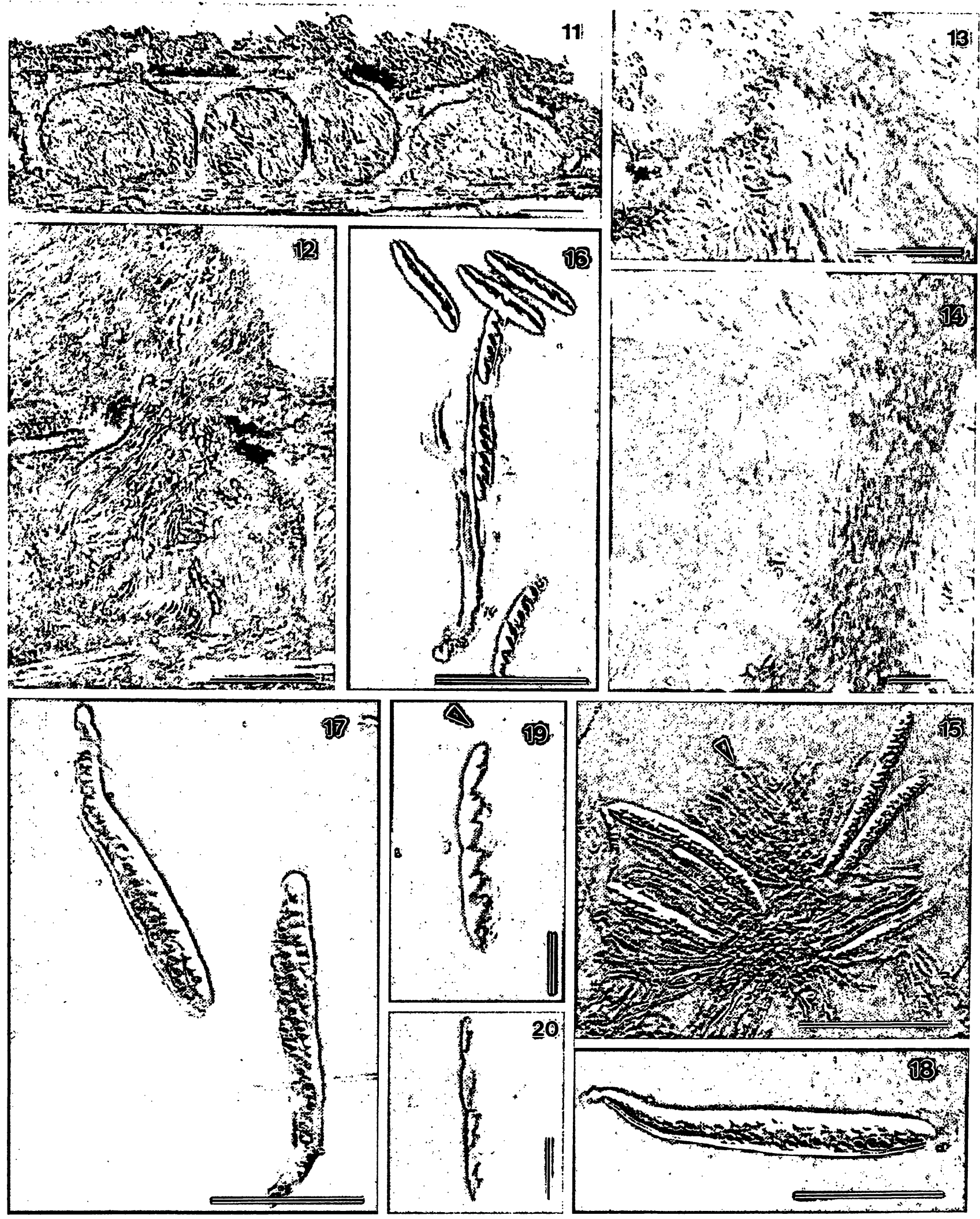

Figs 11-20. Massarina phragmiticola (from holotype).

Fig. 11. Section of aggregated ascomata (bar $=100 \mu \mathrm{m})$. Fig. 12. Section of individual ascoma (bar $=100 \mu \mathrm{m})$. Fig. 13 . Section through papilla (bar $=50 \mu \mathrm{m})$. Fig. 14. Section of peridium (bar $=10 \mu \mathrm{m})$. Fig. 15. Hypha-like septate pseudoparaphyses (arrow) in a gelatinous matrix (bar $=100 \mu \mathrm{m})$. Figs $16-18$. Mature asci (bars $=50 \mu \mathrm{m})$ fissitunicate in 16 . Figs 19-20. Mature ascospores (bars $=10 \mu \mathrm{m}$ ). Fig. 19. Mature ascospore with distal appendage (arrow) markedly smaller than proximal appendage. 


\section{Diagnosis}

Ascomata 180-280 $\mu \mathrm{m}$ in diameter., 150-200 $\mu \mathrm{m}$ alta, solitaria vel aggregata, immersa, subglobosa vel ellipsoidea, nigra, glabra, ostiolata, breve papillata. Asci 102.5-133 $\times 12.5-16.3 \mu \mathrm{m}$, cylindrici vel clavati, fissitunicati, 8-spori, pedicellati. Ascosporae $28-37.5 \times 4.7-6.5 \mu \mathrm{m}(x=33.5 \times 5.3 \mu \mathrm{m}, n=25)$, hyalinae, bicellulares, cylindricae, utrinque obtusae, guttulatae, appendiculatae.

Ascomata $180-280 \mu \mathrm{m}$ in diameter, $150-200 \mu \mathrm{m}$ high, solitary or aggregated, immersed, subglobose to ellipsoidal, black, glabrous, ostiolate, short papillate (Figs 11-13). Peridium 11.8-19.7 $\mu \mathrm{m}$ thick comprising several layers of compressed, brown-walled angular cells (Fig. 14). Pseudoparaphyses ca $85 \mu \mathrm{m}$ long, $2.5-3 \mu \mathrm{m}$ in diameter at the base and $1.5-2.5 \mu \mathrm{m}$ in diameter at the apex, filiform, hypha-like, septate, numerous, mostly free-ended, unbranched or branched at the base, markedly shorter than the asci, invested in mucilage (Fig. 15). Asci 102.5-133 $\times 12.5-16.3 \mu \mathrm{m}$, 8-spored, cylindrical to clavate, fissitunicate, pedicellate, invested in mucilage, developing from base of locule (Figs 15-18). Ascospores 28-37.5 $\times 4.7-6.5 \mu \mathrm{m}$ $(\mathrm{x}=33.5 \times 5.3 \mu \mathrm{m}, \mathrm{n}=25), 2-3$ seriate, 2-celled, hyaline, obtuse at both ends, uni-septate, constricted at septa; apical cell irregularly cylindrical, straight, basal cell cylindrical, straight or slightly curved at the end; guttulate, appendaged at both ends; appendages cupulate, mucilaginous; apical appendage 3.7-6.3 $\mu \mathrm{m}$ in diameter, markedly smaller than basal appendage; basal appendage 7.5 $-9.5 \mu \mathrm{m}$ in diameter (Figs 19, 20). Mode of life: Saprobic.

Habitat: On decaying stems and leaf sheaths of intertidal Phragmites australis.

Known distribution: Hong Kong.

Material examined: Decaying stems and leaf sheaths of Phragmites australis, Mai Po Marshes, Hong Kong $\left(22^{\circ} 29^{\prime} \mathrm{N}, 114^{\circ} 02^{\prime} \mathrm{E}\right), 16$ Nov. 1995, O. K. Poon [HKU(M) 5188, holotype].

Massarina phragmiticola was found to be an infrequent intertidal species occurring on dead $P$. australis stems and leaf sheaths. It can readily be distinguished from the Massarina ricifera Kohlm., Volkm.-Kohlm. et $\mathrm{O}$. Erikss. and $M$. carolinensis Kohlm., Volkm.-Kohlm. et O. Erikss. from Juncus roemerianus (Kohlmeyer et al. 1995 b, 1996) as M. ricifera has smaller ascospores $(19-25 \times 5.5-7 \mu \mathrm{m})$ surrounded by a spreading mucilaginous sheath. In $M$. carolinensis ascospores are also smaller (16.5-21 $\times 4.5-6.5 \mu \mathrm{m})$ and are totally surrounded by a gelatinous sheath. Massarina phragmiticola and $M$. ricifera are marine species, while $M$. carolinensis is a terrestrial species.

Phomatospora phragmiticola $\mathrm{O}$. K. Poon et K. D. Hyde, sp. nov.

Figs 21-35

Etymology: In reference to the host Phragmites australis.

\section{Diagnosis}

Ascomata 155-175 $\mu \mathrm{m}$ in diameter., 125-133 $\mu \mathrm{m}$ alta, immersa, globosa vel subglobosa, membranacea, nigra, ostiolata, breve papillata, solitaria. Asci $85-125$ $\times 5-7.5 \mu \mathrm{m}, 8$-spori, cylindrici, unitunicati, pedicellati, apparatu apicali praediti. Ascosporae 7.5-11.3 $\times 3-5 \mu m(x=9.3 \times 4.2 \mu m ; n=50)$, unicellulares, ellipsoideae, hyalinae, striatae, appendiculatae.

Ascomata $155-175 \mu \mathrm{m}$ in diameter, $125-133 \mu \mathrm{m}$ high, immersed in outer soft tissue of stem, globose to subglobose, membranous, black, ostiolate, short papillate, solitary (Fig. 21). Neck 33-38 $\mu \mathrm{m}$ long, $32-45 \mu \mathrm{m}$ in diameter, cylindrical, papilla central, periphysate (Fig. 22). Peridium 6-7 $\mu \mathrm{m}$ thick, comprising several layers of compressed cells, hyaline inwardly (Fig. 23). Paraphyses 7-8 $\mu \mathrm{m}$ in diameter at the base, hypha-like, straight to flexous, septate, tapering distally, hyaline, numerous, developing from the base of the ascoma (Fig. 24). Asci 85-125 $\times 5-7.5 \mu \mathrm{m}$, eight-spored, cylindrical, unitunicate, thin-walled, pedicellate, with a refractive apical ring which stains in methylene blue, asci developing from base of ascoma (Figs 25-29). Ascospores 7.5-11.3 $\times 3-5 \mu \mathrm{m}(\mathrm{x}=9.3 \times 4.2 \mu \mathrm{m} ; \mathrm{n}=50)$, unicellular, ellipsoidal, hyaline, mostly uniseriate, longitudinally striate, with a bifurcate mucilaginous appendage, 5-8 $\mu \mathrm{m}$ in diameter, provided at each end; appendage does not stain in methylene blue (Figs 30-35).

Mode of life: Saprobic.

Habitat: Stems and leaf sheaths of Phragmites australis.

Known distribution: Hong Kong.

Material examined: Decaying stems and leaf sheaths of intertidal $P$. australis, Mai Po Marshes, Hong Kong $\left(22^{\circ} 29^{\prime} \mathrm{N}, 114^{\circ} 02^{\prime} \mathrm{E}\right), 17$ Aug. 1995 , O. K. Poon [HKU(M) 5189, holotype], decaying stems and leaf sheaths of intertidal $P$. australis, Mai Po Marshes, Hong Kong $\left(22^{\circ} 29^{\prime} \mathrm{N}, 114^{\circ} 02^{\prime} \mathrm{E}\right), 16$ Nov. 1995, O. K. Poon [HKU(M) 5190].

Phomatospora phragmiticola can be considered to be an obligate marine fungus as it has the highest affinity in the intertidal region. Phomatospora phragmiticola can be confused with P. bellaminuta Kohlm., Volkm.Kohlm. et O. E. Erikss. (Kohlmeyer et al. 1995 b) which was found at the lower parts of decaying Juncus roemarianus in the U.S. A. The differences between these species and other marine species are given in Table II. Phragmites phragmiticola differs from $P$. berkelyi Sacc, which has smaller ascospores (Kirk 1984).

Cytoplacosphaeria phragmiticola O. K. Poon et K. D. Hyde, sp. nov.

Figs 36-45

\section{Diagnosis}

Conidiomata ca $800 \mu m$ in diameter, $290 \mu \mathrm{m}$ alta, eustromatica, immersa vel erumpentia, brunnea, ellip- 


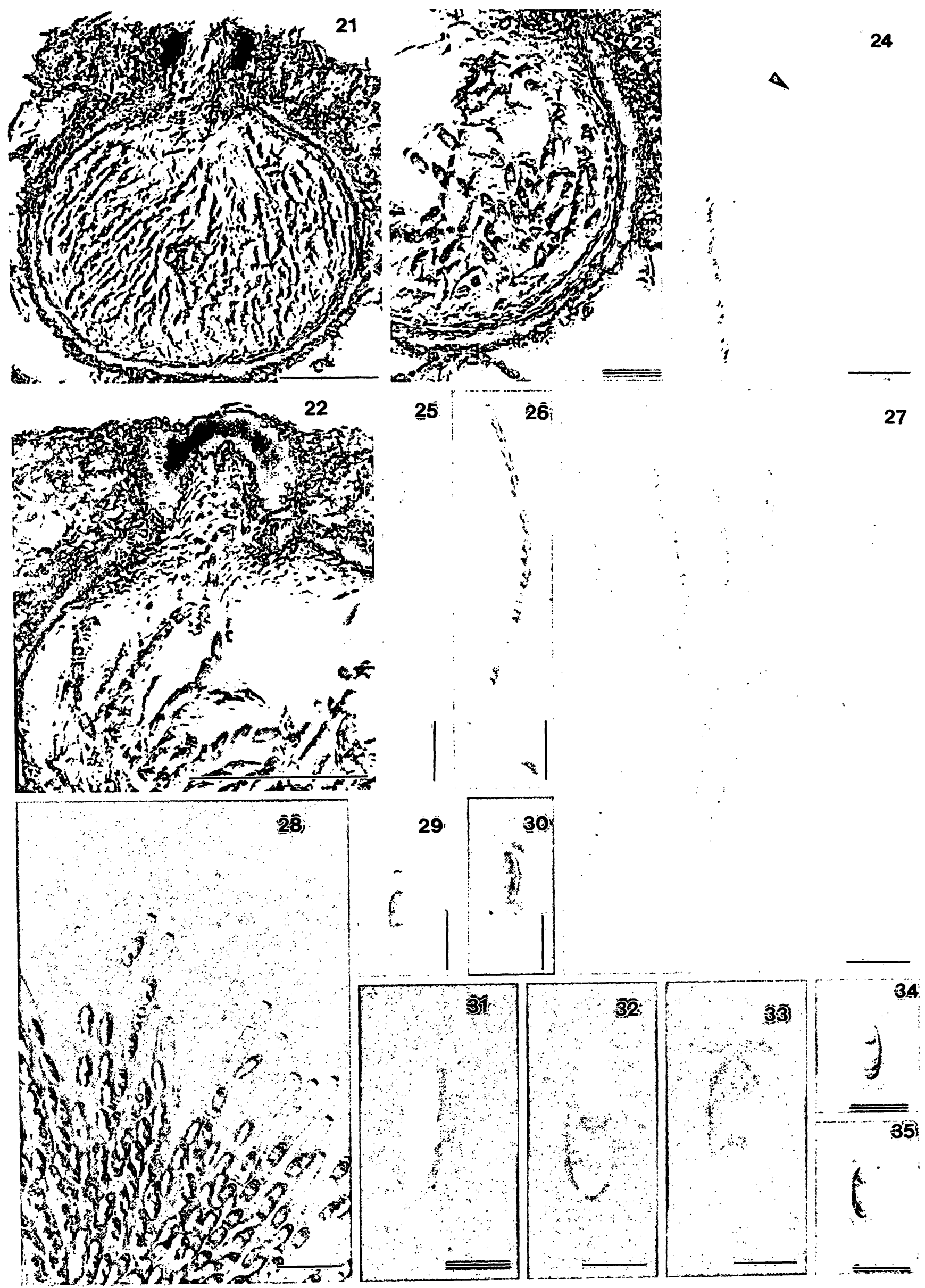

Figs 21-35. Phomatospora phragmiticola (from holotype).

Fig. 21. Section of ascoma (bar $=50 \mu \mathrm{m}$.). Fig. 22. Section through neck (bar $=50 \mu \mathrm{m})$. Fig. 23. Section through peridium (bar $=10 \mu \mathrm{m})$. Fig. 24. Hypha-like hyaline paraphyses $($ bar $=20 \mu \mathrm{m})$. Figs $25-28$. Mature asci $($ bars $=20 \mu \mathrm{m})$. Fig. 29. Apex f mature ascus illustrating apical ring $(\mathrm{bar}=10 \mu \mathrm{m})$. Figs 30,34 and 35. Mature ascospores (bars $=10 \mu \mathrm{m})$. Fig. 39. Mature ascospore, showing lingitudinal striations. Figs $31-33$. Mature ascospores with bifurcate appendages $(\mathrm{bars}=5 \mu \mathrm{m})$. 
Table II. Synopsis of marine species of Phomatospora.

\begin{tabular}{|c|c|c|c|c|}
\hline & $\begin{array}{l}\text { P. acrostichi } \\
\text { K. D. Hyde }\end{array}$ & $\begin{array}{l}\text { P. bellaminuta } \\
\text { Kohlm. et } \\
\text { Volkm.-Kohlm. }\end{array}$ & $\begin{array}{l}\text { P. kandeliae } \\
\text { K. D. Hyde }\end{array}$ & $\begin{array}{l}\text { P. phragmiticola } \\
\text { Kohlm. et } \\
\text { Volkm.-Kohlm. }\end{array}$ \\
\hline Ascomata $(\mu \mathrm{m})$ & $\begin{array}{l}106-274 \text { high } \\
176-330 \text { diam. }\end{array}$ & $\begin{array}{l}115-125 \text { high } \\
125-160 \text { diam. }\end{array}$ & $\begin{array}{l}145-260 \text { high } \\
195-325 \text { diam. }\end{array}$ & $\begin{array}{l}125-133 \text { high } \\
155-175 \text { diam. }\end{array}$ \\
\hline Ascospore size $(\mu \mathrm{m})$ & $5.7-7.1 \times 2-2.8$ & $\begin{array}{l}10.1-12.5(-13) \\
\times 3.7-4.6(\mathrm{x}=11.3 \\
\times 4.1, \mathrm{n}=67)\end{array}$ & $11.5 \times 16 \times 5.5-8$ & $\begin{array}{l}7.5-11.3 \times 3.3-5 \\
(x=9.3 \times 4.2, n=50)\end{array}$ \\
\hline Appendages & $\begin{array}{l}\text { Bifurcate, } \\
\text { at one end }\end{array}$ & $\begin{array}{l}\text { Gelatinous caps, } \\
\text { staining blue in } \\
\text { methylene blue }\end{array}$ & $\begin{array}{l}\text { Caps which may } \\
\text { become filamentous }\end{array}$ & $\begin{array}{l}\text { Bifurcate, not staining } \\
\text { blue in methylene blue }\end{array}$ \\
\hline $\begin{array}{l}\text { Ascospore arrange- } \\
\text { ment in ascus }\end{array}$ & Uniseriate & $1-2$ seriate & $\begin{array}{l}\text { Uni-or-overlapping } \\
\text { uniseriate }\end{array}$ & Uniseriate \\
\hline Ascospore shape & $\begin{array}{l}\text { Ellipsoidal with } \\
\text { rounded ends }\end{array}$ & $\begin{array}{l}\text { Ellipsoidal with } \\
\text { rounded ends }\end{array}$ & $\begin{array}{l}\text { Ellipsoidal with } \\
\text { rounded ends }\end{array}$ & $\begin{array}{l}\text { Ellipsoidal with some- } \\
\text { what acute ends }\end{array}$ \\
\hline Habitat & $\begin{array}{l}\text { Acrostichum, } \\
\text { intertidal }\end{array}$ & $\begin{array}{l}\text { Juncus, lower part } \\
\text { of stems and leaf } \\
\text { sheaths }\end{array}$ & Kandelia, intertidal & $\begin{array}{l}\text { Phragmites, lower parts } \\
\text { of stems and leaf sheats }\end{array}$ \\
\hline Reference & Hyde 1988 & $\begin{array}{l}\text { Kohlmeyer and } \\
\text { Volkmann-Kohlmeyer } \\
\text { 1995 a }\end{array}$ & Hyde 1992 & This paper \\
\hline
\end{tabular}

soidea vel lenticularia. Condiophora nullae. Cellulae conidiogenae 9.8-12.5 $\times 7.3-8 \mu \mathrm{m}$, enteroblasticae, phialidicae, discretae, determinatae, ampulliformes vel doliiformes, laeves. Conidia 17.5-75 × 2.5-5 $\mu \mathrm{m}, \mathrm{cy}$ lindrica, laevia, curva, hyalina, 0-5 septata.

Conidiomata $\mathrm{ca} .800 \mu \mathrm{m}$ in diameter, $290 \mu \mathrm{m}$ high, eustromatic, loosely aggregated into stromata with 1-5 locules, immersed, brown, ellipsoidal to lenticular, scarcely erumpent (Figs 36, 37). Peridium outer wall consists of several layers of thick-walled dark brown cells, in the form of textura angularis; becoming thinner and paler towards the conidiogenous region. Ostioles indistinct, dehiscence possibly by rupture of the upper wall (Fig. 38). Conidiophores absent. Conidiogenous cells 9.8-12.5 $\times 7.3-8 \mu \mathrm{m}$, enteroblastic, phialidic, discrete, determinate, ampulliform to doliiform, smooth, with apical or lateral apertures, collarette clearly visible, channels comparatively wide, hyaline, developing from inner cells of locules (Figs 39-42). Conidia 17.5-75 $\times 2.5-5 \mu \mathrm{m}$, cylindrical, straight, curved or irregular, thin-walled, minutely guttulate, smooth, hyaline, $0-5$ septate, not constricted at septa (Figs 43-45).

Mode of life: Saprobic.

Habitat: On decaying stems and leaf sheaths of Phragmites australis.

\section{Known distribution: Hong Kong.}

Material examined: Decaying stems of leaf sheaths of P. australis, Mai Po Marshes, Hong Kong $\left(22^{\circ} 29^{\prime} \mathrm{N}\right.$, $114^{\circ} 02^{\prime}$ E), 16 Nov. 1995, O. K. Poon [HKU(M) 5191, holotype].
Cytoplacosphaeria phragmiticola is a facultative brackish water species frequently occurring on Phragmites australis stems and leaf sheaths collected from the sides of the gei wai. It has a high affinity around 10-20 $\mathrm{cm}$ above the water-air interface, which is the upper to middle portion of $P$. australis stems and leaf sheaths. Cytoplacosphaeria phragmiticola was recovered once on the basal portion of $P$. australis stem and leaf sheath in the intertidal region outside the gei wai.

This species is similar to the type species Cytoplacosphaeria rimosa (oud.) Petr., which occurs on stems of leaf sheaths of Phragmites australis, $P$. vulgaris Crepin and Phragmites sp., in Latvia, Czechoslovakia and England (Sutton 1980). However, C. rimosa has conidiogenous cells with a distinct collarette and shorter eguttulate conidia $(13-20 \times 3 \mu \mathrm{m})$. This makes $C$. phragmiticola readily distinguishable from $C$. rimosa. The ostiole in $C$. rimosa is reported to be single, circular and papillate, whereas it appears that C. phragmiticola dehisces by rupture of the upper wall.

\section{Microsphaeropsis sp.}

Figs 46-55

Conidiomata pycnidial, ampulliform, occasionally subglobose, black, immersed, papillate, mostly unilocular, occasionally bilocular, ca $280 \mu \mathrm{m}$ in diameter, $240 \mu \mathrm{m}$ high (Figs 46, 47). Peridium 11-23 $\mu \mathrm{m}$ thick, outer wall of $1-3$ layers of thick-walled dark brown cells, in the form of textura angularis; middle wall of 3-5 layers of thick-walled pale brown cells, 

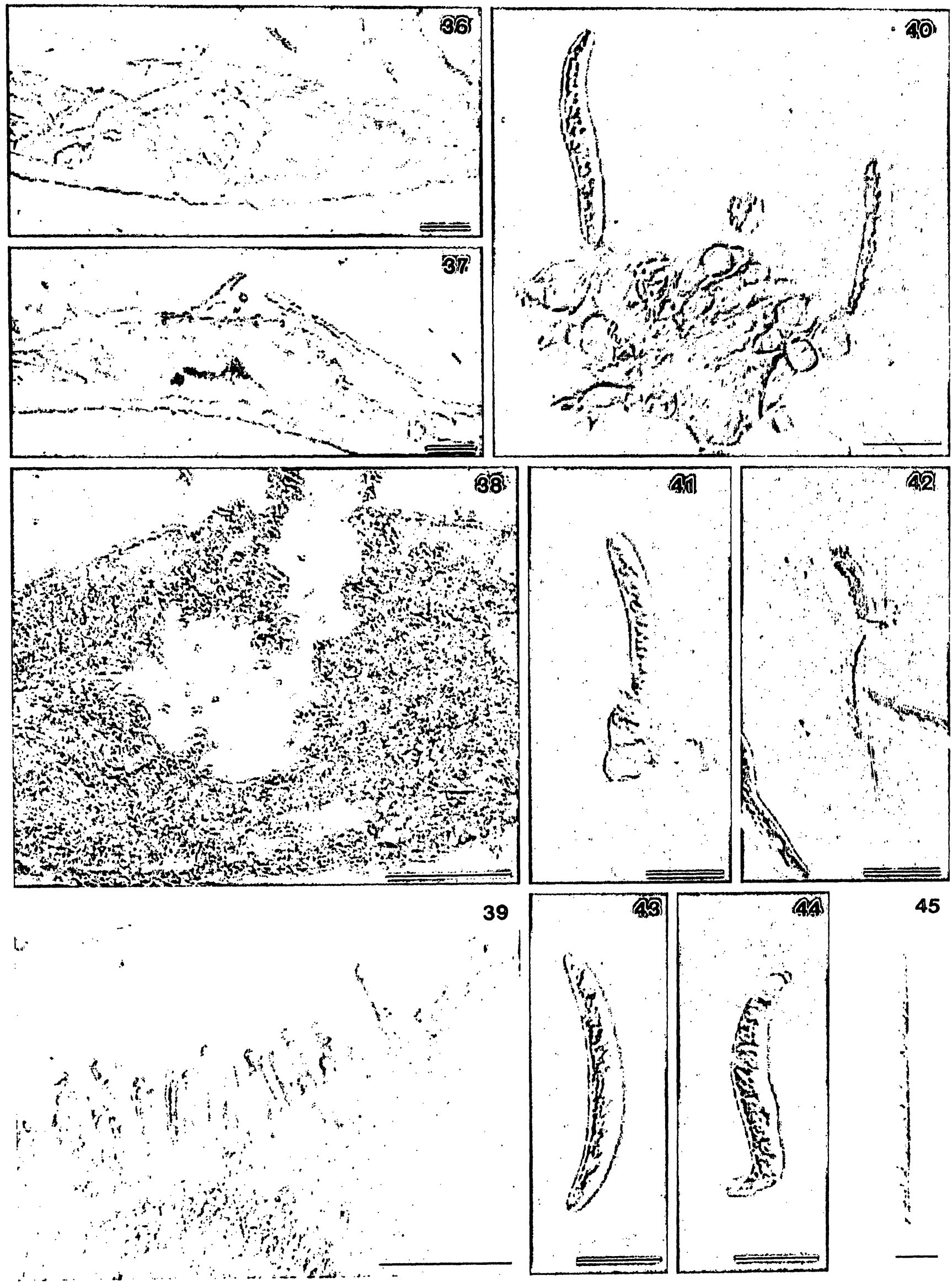

Figs 36-45. Cytoplacosphacita phragmiticola (from hololypc).

Figs 36-38. Sections of conidiomatil (bars $=100 \mu \mathrm{m})$. Fig. 39. Conidia arising from conidiogenous cells developing from basc of loculc (bar $=50 \mu \mathrm{m}$. Figs 40,41 . Conidiognous cells with developing conidia (bars $=20 \mu \mathrm{m}$ ). Fig. 42. Conidingenous cell (bar $=20 \mu \mathrm{m})$. Figs 43-55. Mature conidia (barts $=5 \mu \mathrm{m})$. 


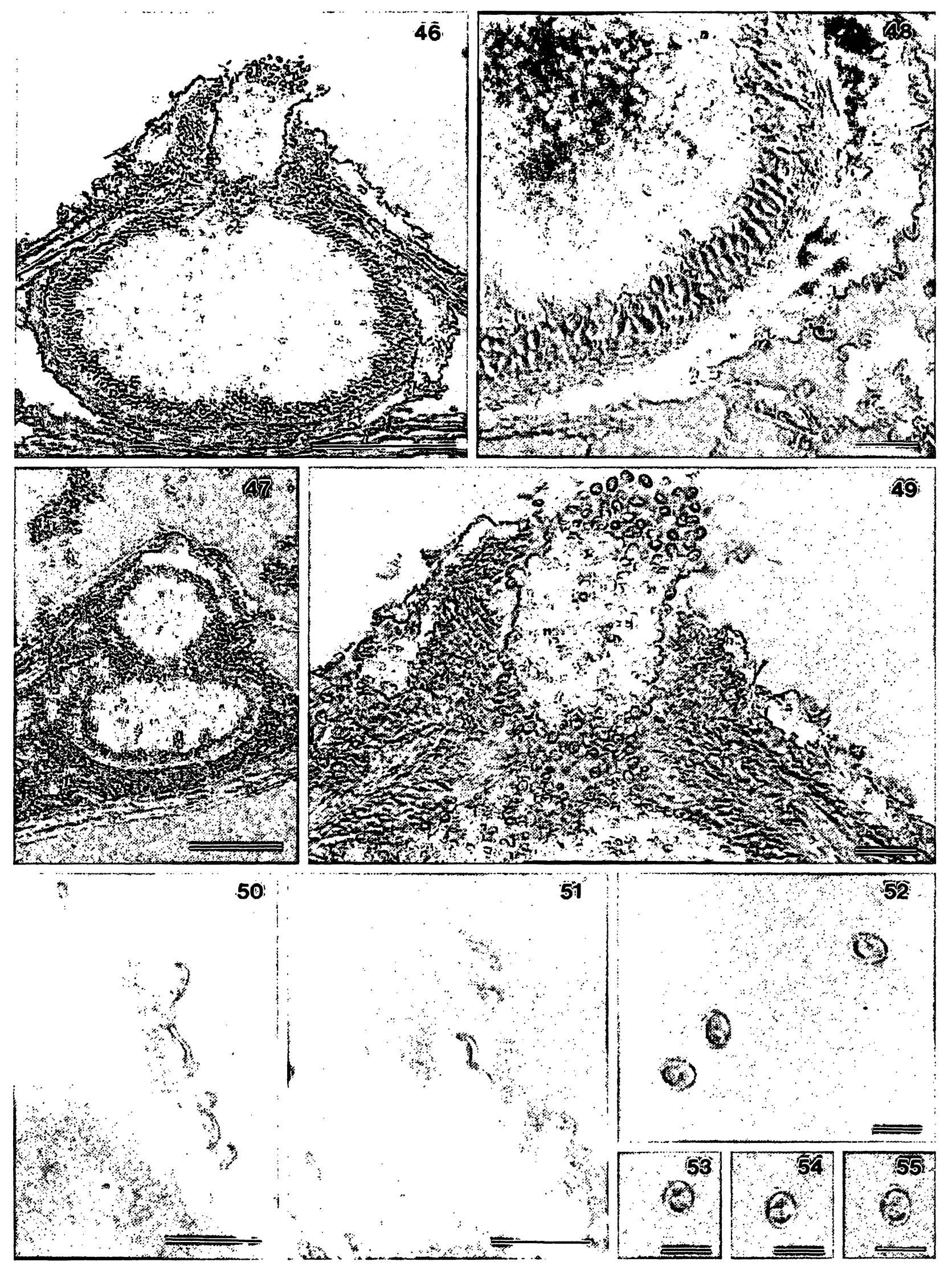

Figs 46-55. Microsphaeropsis sp.

Figs 46, 47. Sections of conidiomata (bars $=100 \mu \mathrm{m})$. Fig. 48. Section through peridium (bar $=20 \mu \mathrm{m})$. Fig. 49 . Section through papilla $(\mathrm{bar}=20 \mu \mathrm{m})$. Figs 50,51 . Condiophores with developing conidia (bars $=10 \mu \mathrm{m})$. Figs $52-55$. Mature conidia (bars $=5 \mu \mathrm{m})$. 
in the form of textura porrecta; inner wall of single layer of hyaline thin-walled cells, in the form of textura angularis (Fig. 48). Papilla ca $80 \mu \mathrm{m}$ long, 130 $\mu \mathrm{m}$ wide (Fig. 49). Conidiophores absent. Conidiogenous cells enteroblastic, phialidic, determinate, discrete, doliiform to cylindrical, collarette minute, smooth, hyaline, developing from inner cells of pycnidial wall (Figs 50, 51). Conidia 3.7-5 × 3.7$4.5 \mu \mathrm{m}, 1$-celled, brown, thick-walled, ellipsoidal, biguttulate at both ends, aseptate, enclosed in a thin mucilagenous sheath (Figs 52-55).

This is a marine species which occurred only at the basal portion of decaying standing stems and leaf sheaths of Phragmites australis and on decaying stems and leaf sheaths of $P$. australis submerged in brackish water. No species from this genus is reported on salt marsh plants. There are 800 names in Coniothyrium and many of them probably belong in Microsphaeropsis (Sutton 1980). A major revision of the group is needed and therefore it would be unwise to name this species beyond generic level. Coniothyrium obiones Jaap is a marine species which has been reported on dead stems and leaf sheaths and moribund propagules of a coastal salt marsh plant Halimione portulacoides (L.) Aellen collected in England and Germany (Kohlmeyer and Kohlmeyer 1979). Microsphaeropsis sp. collected from Phragmites australis, has non-tapered conidiogenous cells and conidia enclosed in mucilaginous sheaths, and differs from Coniothyrium obiones Jaap.

\section{Discussion}

\section{Biodiversity of fungi found in Hong Kong mangroves}

The results of these studies are preliminary observations on the fungi involved in the decay of stems and leaf sheaths of Phragmites australis at Mai Po Marshes in Hong Kong. Because of the relatively small sample sizes based on two site visits in August and November 1995 the data on frequency and composition must be treated with caution as additional collections at different times may result in different compositions. However, the results do represent the first data on the biodiversity of fungi on grasses in the tropics.

Similar studies on biodiversity of fungi on other grasses in Hong Kong are unavailable for comparison. However, there have been several reports on lignicolous fungi from mangroves in Hong Kong (Vrijmoed 1990, Vrijmoed et al. 1994, Sadaba et al. 1995).

In the study of Vrijmoed (1990), Vrijmoed et al. (1994) and Sadaba et al. (1994), and in this study, Lignincola laevis was the most frequently identified fungus on Acanthus ilicifolius L., Avicennia marina, Kandelia candel, Phragmites australis and driftwood. Aniptodera chesapeakensis was also commonly found on Acanthus ilicifolium, Avicennia marina and Phragmites australis. Halosarpheia sp. and Lulworthia sp. were common to both Kandelia candel and driftwood. Species of Leptosphaeria were found on Acanthus ilicifolium and Phragmites australis and driftwood. Halonectria milfordensis was found on Aegiceras corniculatum and Kandelia candel.

The number of taxa recorded in this study (61) is higher than reported by Sadaba et al. (1995) on Acanthus ilicifolium. Only 44 higher fungi were found associated with decaying standing parts of Acanthus ilicifolium (Sadaba et al. 1995). There were differences in fungal composition on Phragmites australis and Acanthus ilicifolium. In the intertidal region of Mai Po Marshes, the most common taxa on standing parts of Acanthus ilicifolium were Acremonium sp. (55\%), Colletotrichum sp. (42.5\%), Phoma sp. (42.5\%), Fusarium sp. (25\%) and Tubercularia sp. (24.2\%) (Sadaba et al. 1995). On the other hand, the common taxa found on dead stems and leaf sheaths of Phragmites australis were Lignincola laevis (22.7\%), Colletotrichum sp. (21.2\%), Phomopsis sp. 1 (19.3\%), Aniptodera phragmiticola (15.2\%), Fusarium sp. (14.9\%), Cladosporium sp. (13\%) and Trichoderma sp. $(12.3 \%)$.

The differences in mycota reported may be due to different host species studied. Sadaba et al. (1995) only collected Acanthus ilicifolium samples from the intertidal region of Mai Po Marshes (outsides the security fence), while Phragmites australis were collected from both the intertidal region and along the edge of the gei wai. There appears to be a group of fungi which are only found inside or along the edge of the gei wai. Nonetheless, two frequent taxa (Colletotrichum sp. and Fusarium sp.) are common to both hosts.

Few mitosporic fungi are reported by Vrijmoed (1990), since most mitosporic fungi are terrestrial species and are unlikely to occur on the decaying plant samples found in the aquatic environment. The large numer of fungi recorded from Phragmites australis and Acanthus ilicifolius reflected the intensive study of these plants. Further study is needed concerning the host specificity of fungi associated with mangrove plants in Hong Kong.

\section{Biodiversity of salt marsh fungi found on grasses}

The diversity of fungi recorded from other salt marsh grasses, such as species of Spartina is lower than those found in this study. Phragmites anstralis has the greatest number of fungal species recorded (63), followed by Spartina alterniflora (49), Spartina townsendii $\mathrm{H}$. Groves et J. Groves (39), an unidentified species of Spartina (30), Spartina patens (Aiton.) Muhl. (16), Spartina cynosuroides (L.) Roth (14), Spartina foliosa Trin. (3), Spartina maritima (Curtis) Fernald (2), and Spartina anglica C. E. Hubbard (1) (Gessner and Kohlmeyer 1976).

The most common species found on these species of Spartina are Buergenerula spartinae Kohlm. et 
Gessner, Claviceps purpurea (Fr.) Tul., Phaeosphaeria albopunctata, Leptosphaeria marina Ellis et Everh., Passeriniela obiones (Crouan et Crouan) K. D. Hyde et Mouzouras, species of Lulworthia, Phaeosphaeria spartinicola Leucht., Puccinia sparganioides. Ellis et Barth., Phoma sp., and Stagonospora sp. (Gessner and Kohlmeyer 1976). The most common taxa on Phragmites australis are Aniptodera phragmiticola, Cladosporium sp., Colletotrichum sp., Fusarium sp., Lignincola laevis, Phomopsis sp. 1 and Trichoderma sp.

Gessner (1977) found a characteristic group of fungi associated with Spartina alterniflora along the east coasts of North and South America. Differences in fungal taxa found on Phragmites australis and species of Spartina can be attributed to temperature, host specificity and location. Perhaps it is unlikely for a group of fungi adapted to decomposing $S$. alterniflora to be found on decaying stems and leaf sheaths of $P$. australis. Further investigation is required regarding host specificity of saprophytic fungi on grasses.

\section{Saprophytic fungi occurring on Phragmites australis in Mai Po}

The differences in fungal composition at the two sites in Mai Po may be due to the preference of fungi towards certain inundation periods. Salinity difference is not a factor contributing to these differences. Salinities in intertidal region and gei wai are nearly the same due to the connection of two water bodies via the sluice gate. There are also differences in species composition of terrestrial fungi between the two sites. This is not expected since terrestrial fungi are not affected by the differences of inundation period as in aquatic species.

There are also differences between the intertidal and terrestrial fungi. With the exception of Chaetomium globosum all ascomycetes are intertidal fungi (e. g. Phomatospora phragmiticola and Lignincola laevis). On the other hand, most terrestrial taxa were mitosporic fungi, such as Fusarium sp., Gliomastix sp. 2. sp., Macrophomina sp. and Trichoderma sp. This may be due to the fact that mitosporic fungi, especially hyphomycetes are better adapted to the terrestrial environment at the upper level of the dead standing plant. Rapid colonisation of substrates by production of large amount of light conidia is characteristic of most hyphomycetes.

Terrestrial fungi (e.g. Chaetomium globosum and Dendrostilbella $\mathrm{sp}$.) were not recovered from lower portions of standing decaying stems and leaf sheaths of Phragmites australis as they are probably not adapted to periodic submergence in seawater. This was also observed in the succession pattern of decomposers on $P$. australis in Japan (Tanaka 1991).

In conclusion, fungal communities associated with decaying Phragmites australis permanently submerged in the gei wai, are different to those in the intertidal region. The diversity of fungal taxa found in this study is probably an underestimate, since the identification of species depends on the reproductive structures being produced, and a relatively short study was carried out.

We would like to thank Dr T. K. Goh for identifying some of the mitosporic fungi listed in this study. Professor J. Kohlmeyer and Dr T. K. Goh are thanked for presubmission reviews. H. Leung and A. Y. P. Lee are thanked for technical assistance.

Accepted 20 August 1997

\section{References}

Cunnell, G. J. 1958. On Robillarda phragmitis sp. nov. Trans. Br. Mycol. Soc. 41: 405-412.

Gessner, R. V. 1976. In vitro growth and nutrition of Buergenerula spartinae, a fungus associated with Spartina alterniflora. Mycologia 68: 583-599.

Gessner, R. V. 1977. Seasonal occurrence and distribution of fungi associated with Spartina alterniflora from a Rhode Island estuary. Mycologia 69: 477-491.

Gessner, R. V. and J. Kohlmeyer. 1976. Geographical distribution and taxonomy of fungi from salt marsh Spartina. Can. J. Bot. 54: 2023-2037.

Hyde, K. D. 1988. Phomatospora acrostochi. sp. nov., a marine fungus on pinnae of Acrostichum speciosum. Trans. Brit. Mycol. Soc. 90: 135-138.

Hyde, K. D. 1992. Intertidal fungi from Kandelia candel including Phomatosporá kandeliae sp. nov. Trans Mycol. Soc. Japan 33: 313-316.

Hyde, K. D. and E. B. G. Jones. 1988. Marine mangrove fungi. Mar. Ecol. (P.S.Z. N. I.) 9: 15-33.

Hyde, K. D. and A. Nakagiri. 1992. Intertidal fungi from Australia. The genus Swanpomyces including S. triseptatus sp. nov. Sydowia 44: 122-130.

Hyde, K. D., A. Chalermpongse and T. Boonthavikoon. 1993. The distribution of intertidal fungi on Rhizophora apiculata. In: (B. Morton, ed.) The Marine Biology of the South China Sea. Hong Kong University Press, Hong Kong. pp. 643-652.

Hyde, K. D., C. A. Farrant and E. B. G. Jones. 1986. Marine fungi from Seychelles III. Aniptodera mangrovii $s p$. nov. from mangrove wood. Can. J. Bot. 64: 2989-2992.

Hyde, K. D., L. P. Vrijmoed, S. Chinnaraj and E. B. G. Jones. 1992. Massarina armatispora sp. nov., a new intertidal ascomycetes from mangroves. Bot. Mar. 35: 325328.

Johnson, T. W. and F. K. Sparrow Jr. 1961. Fungi in Oceans and Estuaries. J. Cramer. Weinheim, Germany.

Jones, E. B. G. and R. Agerer. 1992. Calathella mangrovei sp. nov. and observations on the mangrove fungus Halocyphina villosa. Bot. Mar. 35: 259-265. 
Karsten, P. A. 1873. Mycologia Fennica. 2. Bidr. t. Kännedom. af Finlands Natur och Folk, Helsinfors 1-252.

Kirk, P. M. 1984. New or interesting microfungi. XIII. Ascomycetes on Laurus nobilis leaf litter. Mycotaxon 19: 307-322.

Kohlmeyer, J. and E. Kohlmeyer. 1979. Marine Mycology, the Higher Fungi. Academic Press, New York. 690 pp.

Kohlmeyer, J. and B. Volkmann-Kohlmeyer. 1993 a. Two new genera of Ascomycotina from saltmarsh Juncus. Syst. Ascom. 11: 95-106.

Kohlmeyer, J. and B. Volkmann-Kohlmeyer. 1993 b. Atrotorquata and Loratospora: New ascomycete genera on Juncus roemarianus. Syst. Ascom. 12: 7-22.

Kohlmeyer, J. and B. Volkmann-Kohlmeyer. 1995. Fungi on Juncus roemerianus. I. Trichocladium medullare. $M y$ cotaxon 53: 349-353.

Kohlmeyer, J., B. Volkmann-Kohlmeyer and O. E. Eriksson. 1995 a. Fungi on Juncus roemerianus. 2. New dictyosporous ascomycetes. Bot. Mar. 38: 165-174.

Kohlmeyer, J., B. Volkmann-Kohlmeyer and O. E. Eriksson. 1995 b. Fungi on Juncus roemerianus. 3. New ascomycetes. Bot. Mar. 38: 175-186.

Kohlmeyer, J., B. Volkmann-Kohlmeyer and O. E. Eriksson. 1995 c. Fungi on Juncus roemerianus. 4. New marine ascomycetes. Mycologia 87: 532-542.

Kohlmeyer, J., B. Volkmann-Kohlmeyer and O. E. Eriksson. 1996. Fungi on Juncus rocmerianus. 5. New marine and terrestrial ascomycetes. Mycol. Res. 100: 393-404.

Lee, S. Y. 1990. Net aerial primary productivity, litter production and decomposition of the reed Phragmites australis in a nature reserve in Hong Kong: management implications. Mar. Ecol. Prog. Ser. 66: 161-173.

Macintosh, D. J. 1983. Fisheries and aquaculture significance of mangroves swamps, with special reference to the Indo-West pacific region. In: (J. F. Muir and R. J. Roberts, eds.) Recent Advances in Aquaculture. Croom Helm, London. pp. 5-85.

Meyers, S. P. 1974. Contribution of fungi to the biodegradation of Spartina and other brackish marshland vegetation. Veroeff. Inst. Meeresforsch. Bremerhaven Suppl. 5: $357-375$.
Meyers, S. P., M. L. Nicholson, J. Rhee, P. Miles and D. G. Ahearm. 1970. Mycological studies in Barataria Bay, Louisiana, and biodegradation of oyster grass, Spartina alterniflora, Louisiana State Univ. Coastal Stud. Bull. 5: 111- 124 .

Newell, S. Y. 1993. Decomposition of shoots of a salt marsh grass. Advances in Microbioal Ecology 13: 301-326.

Newell, S. Y. 1996. Established and potential impacts of eukaryotic mycelial decomposers in marine/terrestrial ecotones. J. Exp. Mar. Biol. Ecol. 200: 187-206.

Sadaba, R. B., L. P. Vrijmoed, E. B. G. Jones and I. J. Hodgkiss. 1995. Observations on vertical distribution of fungi associated with standing decaying Acanthus ilicifolius stems at Mai Po Mangrove, Hong Kong Hydrobiologia 295: 119-126.

Sutton, B. C. 1980. The Coelomycetes. Commonwealth Mycological Institute. $696 \mathrm{pp}$.

Sutton, B. C. and J. L. Alcorn. 1974. Neottiosporina. Aust. J. Bot. 22: 517-530.

Tan, T. K. and W. F. Leong. 1989. Succession of fungi on wood of Avicennia alba A. lanata in Singapore. Can. J. Bot. 67: 2686-2691.

Tanaka, Y. 1991. Microbial decomposition of reed (Phragmites australis) leaves in a saline lake. Hydrobiologia 220: $119-129$

Volkmann-Kohlmeyer, B. and J. Kohlmeyer. 1993. Atrotorquata and Loratospora: New Ascomycete genera on Juncus roemerianus. Syst. Ascom. 12: 7-21.

Volkmann-Kohlmeyer, B. and J. Kohlmeyer. 1994. A new Aniptodera (Ascomycotina) from saltmarsh Juncus. Bot. Mar. 37: 109-114.

Vrijmoed, L. P. 1990. Preliminary observations of lignicolous marine fungi from mangroves in Hong Kong. In: (B. Morton, ed.) Proceedings of the Second International Marine Biological Workshop: The Marine Flora and Fauna of Hong Kong and Soulhern China. Hong Kong University Press, Hong Kong. pp. 701-706.

Vrijmoed, L. P., E. B. G. Jones and K. D. Hyde. 1994. Observations on subtropical mangrove fungi in the Pearl River Estuary. Acta Scientiarum Naturalium Universitatis Sunyatseni 33: 78-85. 
